\title{
Theory of the Linewidth of Intersubband Plasmons in Quantum Wells
}

\author{
C. A. Ullrich ${ }^{1,2}$ and G. Vignale ${ }^{2}$ \\ ${ }^{1}$ iQUEST, University of California, Santa Barbara, California 93106 \\ ${ }^{2}$ Department of Physics, University of Missouri, Columbia, Missouri 65211
}

(Received 14 December 2000; published 2 July 2001)

\begin{abstract}
Intersubband (ISB) plasmons in remotely doped wide quantum wells acquire a linewidth even at zero temperature and in-plane wave vector $q_{\|}=0$ by a combination of intrinsic (electron-electron interaction) and extrinsic effects (impurities and interface roughness). We present a quantitatively accurate theory of the linewidth that treats both effects on equal footing and from first principles by a combination of time-dependent density-functional theory with the memory function formalism. Comparison with recent optical absorption experiments shows that the ISB plasmon linewidth has a significant contribution from electron-electron interaction, and is only weakly related to the mobility.
\end{abstract}

DOI: 10.1103/PhysRevLett.87.037402

In semiconductor quantum wells, the conduction band splits up into several subbands, and electrons (supplied, e.g., by remote doping) can perform collective transitions between them. These so-called intersubband (ISB) plasmons are currently of great experimental and theoretical interest [1], and they are the basis of a variety of new devices operating in the terahertz regime, such as detectors [2] and quantum cascade lasers [3]. In designing these devices, the emphasis usually lies in covering a particular frequency range. However, often it is desirable that the transitions also have a narrow linewidth, to achieve better frequency resolution and larger peak absorption in detectors, and higher gain in lasers. The linewidth arises from a complicated interplay of a variety of scattering mechanisms, intrinsic (electron-electron and electron-phonon) as well as extrinsic ones (impurity, alloy disorder, and interface roughness). Many aspects of this interplay are still not well understood, in particular the relative importance of the individual mechanisms [4].

To disentangle the various contributions to the ISB linewidth, it is helpful to consider a situation where some of them are not effective. In a recent experiment, Williams et al. [5] studied collective ISB transitions in an $n$-type 40 -nm-wide single $\mathrm{GaAs} / \mathrm{Al}_{0.3} \mathrm{Ga}_{0.7} \mathrm{As}$ quantum well, with Si doping centers $100 \mathrm{~nm}$ away from the well. Sharp transitions were found well below the LO phonon frequency of GaAs $(35.6 \mathrm{meV})$, at a temperature of $2.3 \mathrm{~K}$. Thus, neither remote impurity nor phonon scattering play any significant role (nor is alloy-disorder scattering, as shown in [6]). The linewidth is therefore expected to be dominated by bulk impurity and interface roughness scattering, while electronic many-body effects have traditionally been neglected. However, for high-quality samples this is no longer justified. In this Letter, we present a theory that treats both damping mechanisms on equal footing, by combining time-dependent density-functional theory [7] (which includes purely electronic damping [8-10] but neglects disorder) with the memory function formalism [11-13] (which describes disorder scattering, but ignores electronic damping).
PACS numbers: 78.67.De, 71.15.Mb, 71.45.Gm, 73.21.Fg

In the experiment [5], two parameters were controlled independently: the electronic sheet density $N_{s}$ (from $0.05 \times 10^{11}$ to $1.3 \times 10^{11} \mathrm{~cm}^{-2}$ ), and a static electric field $E$ perpendicular to the well which pushes the electrons against one of its edges. This provides an ideal tool to distinguish interface roughness from other damping effects.

ISB plasmons will be described using linear response theory within a one-band effective-mass approximation (units are chosen such that $e^{*}=m^{*}=\hbar=1$, where $e^{*}$ and $m^{*}$ are the effective charge and mass in GaAs). The density response to a frequency-dependent external perturbation $v_{\text {ext }, 1}(\mathbf{r}, \omega)$ is given by

$$
n_{1}(\mathbf{r}, \omega)=\int d^{3} r^{\prime} \chi\left(\mathbf{r}, \mathbf{r}^{\prime}, \omega\right) v_{\mathrm{ext}, 1}\left(\mathbf{r}^{\prime}, \omega\right) \text {. }
$$

All damping effects are contained in the response function $\chi\left(\mathbf{r}, \mathbf{r}^{\prime}, \omega\right)$ of the system, discussed below in detail. We consider perturbations of the form $v_{\text {ext }, 1}(z, \omega)=E_{0} z$, corresponding to monochromatic plane electromagnetic waves of amplitude $E_{0}$ polarized along the $z$ axis, the direction of growth of the quantum well. Having solved the response equation (1), the photoabsorption cross section is obtained as $\sigma(\omega)=-\left(8 \pi \omega / E_{0} c\right) \operatorname{Im} \int d z z n_{1}(z, \omega)$, which can be directly compared with data from photoabsorption measurements. $\sigma(\omega)$ has a peak at the plasmon frequency $\Omega$ with linewidth (HWHM) $\Gamma$.

The remaining problem is to calculate the response function $\chi$. Let us first consider a "clean" quantum well, in the absence of any extrinsic damping. This case was treated in detail in Ref. [10] using time-dependent density-functional theory, and we summarize here only the key aspects. The full response function of a clean interacting system, $\chi_{c}$, can be exactly written as [7]

$$
\begin{aligned}
\chi_{c}^{-1}\left(\mathbf{r}, \mathbf{r}^{\prime}, \omega\right)= & \chi_{0}^{-1}\left(\mathbf{r}, \mathbf{r}^{\prime}, \omega\right)-\frac{1}{\left|\mathbf{r}-\mathbf{r}^{\prime}\right|} \\
& -f_{\mathrm{xc}}\left(\mathbf{r}, \mathbf{r}^{\prime}, \omega\right) .
\end{aligned}
$$

Here, $\chi_{0}$ is the response function for noninteracting electrons which reads, in a mixed representation $\left(\mathbf{q}_{\|}\right.$is the wave 
vector in the $x-y$ plane of the quantum well),

$$
\begin{aligned}
\chi_{0}\left(\mathbf{q}_{\|}, z, z^{\prime}, \omega\right)= & \sum_{\mu=1}^{N_{\text {occ }}} \sum_{\nu=1}^{\infty} F_{\mu \nu}\left(q_{\|}, \omega\right) \varphi_{\mu}(z) \varphi_{\mu}\left(z^{\prime}\right) \\
& \times \varphi_{\nu}(z) \varphi_{\nu}\left(z^{\prime}\right),
\end{aligned}
$$

where

$$
\begin{aligned}
F_{\mu \nu}\left(q_{\|}, \omega\right)= & -2 \int \frac{d^{2} k_{\|}}{(2 \pi)^{2}} \\
& \times\left\{\frac{f\left(\epsilon_{\mu}+k_{\|}^{2} / 2\right)}{\mathbf{q}_{\|} \mathbf{k}_{\|}+a_{\mu \nu}\left(q_{\|}\right)+\omega+i \eta}\right. \\
& \left.+\frac{f\left(\epsilon_{\mu}+k_{\|}^{2} / 2\right)}{\mathbf{q}_{\|} \mathbf{k}_{\|}+a_{\mu \nu}\left(q_{\|}\right)-\omega-i \eta}\right\},
\end{aligned}
$$

$a_{\mu \nu}\left(q_{\|}\right)=q_{\|}^{2} / 2+\epsilon_{\nu}-\epsilon_{\mu}, f$ is the Fermi function at $T=0$, and $\eta$ is a positive infinitesimal. $\epsilon_{\mu}$ and $\varphi_{\mu}(z)$ are the Kohn-Sham energies and wave function [in localdensity approximation (LDA)] of the quantum well. For the experimental range of $N_{s}$, the system under study has nine bound levels, only the lowest being occupied $\left(N_{\text {occ }}=1\right)$ [10]. The exchange-correlation (xc) kernel $f_{\mathrm{xc}}\left(\mathbf{r}, \mathbf{r}^{\prime}, \omega\right)$ accounts, in principle exactly, for the difference between the full and the RPA response function and has to be approximated in practice. Widely used is the adiabatic local-density approximation (ALDA):

$$
f_{\mathrm{xc}}^{\mathrm{ALDA}}\left(\mathbf{r}, \mathbf{r}^{\prime}, \omega\right)=\left.\delta\left(\mathbf{r}-\mathbf{r}^{\prime}\right) \frac{d^{2} e_{\mathrm{xc}}^{\mathrm{hom}}(\bar{n})}{d \bar{n}^{2}}\right|_{\bar{n}=n(\mathbf{r})},
$$

where $e_{\mathrm{xc}}^{\mathrm{hom}}(\bar{n})$ is the xc energy density of the homogeneous electron gas, and $n(\mathbf{r})$ is the ground-state density.

ISB plasmon dispersions $\Omega\left(q_{\|}\right)$in quantum wells have been widely studied [14]. The imaginary part of $\chi_{0}$ determines the region of damping by single-particle excitations (Landau damping). Outside that region, in particular at small $q_{\|}$, ISB plasmons are undamped in ALDA, since $f_{\mathrm{xc}}^{\mathrm{ALDA}}$ is frequency independent and real. In reality, however, the absence of momentum conservation in the $z$ direction and coupling via Coulomb interaction opens the possibility of plasmon decay into more complicated excitations, such as multiple electron-hole pairs, even at $q_{\|}=0$. To take this effect into account, one has to go beyond the ALDA and include dynamical xc effects.

For this purpose, we consider the linear current density response $\mathbf{j}_{1}(\mathbf{r}, \omega)$ to an external vector potential. $\mathbf{j}_{1}(\mathbf{r}, \omega)$ can be expressed, in principle exactly, as the current response of a noninteracting system to an effective vector potential. The latter has an xc part whose local-density approximation [9] is given by

$$
\begin{aligned}
i \omega a_{\mathrm{xc}, 1, \alpha}(\mathbf{r}, \omega)= & \nabla_{\alpha} v_{\mathrm{xc}, 1}^{\mathrm{ALDA}}(\mathbf{r}, \omega) \\
& -\frac{1}{n(\mathbf{r})} \sum_{\beta} \nabla_{\beta} \sigma_{\mathrm{xc}, \alpha \beta}(\mathbf{r}, \omega),
\end{aligned}
$$

where $\alpha, \beta$ are Cartesian components, and $v_{\mathrm{xc}, 1}(\mathbf{r}, \omega)=$ $\int f_{\mathrm{xc}}\left(\mathbf{r}, \mathbf{r}^{\prime}, \omega\right) n_{1}\left(\mathbf{r}^{\prime}, \omega\right)$. The dynamical correction to the
ALDA features the viscoelastic stress tensor

$$
\begin{aligned}
\sigma_{\mathrm{xc}, \alpha \beta}= & \eta_{\mathrm{xc}}\left(\nabla_{\beta} u_{1, \alpha}+\nabla_{\alpha} u_{1, \beta}-\frac{2}{3} \nabla \cdot \mathbf{u}_{1} \delta_{\alpha \beta}\right) \\
& +\zeta_{\mathrm{xc}} \nabla \cdot \mathbf{u}_{1} \delta_{\alpha \beta} .
\end{aligned}
$$

Here, $\mathbf{u}_{1}(\mathbf{r}, \omega)=\mathbf{j}_{1}(\mathbf{r}, \omega) / n(\mathbf{r})$ is the velocity field, and $\eta_{\mathrm{xc}}(\omega, n(\mathbf{r}))$ and $\zeta_{\mathrm{xc}}(\omega, n(\mathbf{r}))$ are complex viscosity coefficients whose explicit form is given in Ref. [9]. For $\mathbf{q}_{\|}=$ $0, f_{\mathrm{xc}}\left(z, z^{\prime}, \omega\right)$ can be explicitly extracted from $a_{\mathrm{xc}, 1}(z, \omega)$ [10]. Inversion of Eq. (2) then yields $\chi_{c}\left(z, z^{\prime}, \omega\right)$.

In Fig. 1 we show the electric field dependence of the ISB plasmon frequencies $\Omega$ for different values of $N_{s}$. In the experimental data, built-in electric fields are subtracted, so that $\Omega(E)$ exhibits a minimum for $E=0$ and rises quadratically for small fields. $\Omega(E)$ increases most rapidly for the smallest $N_{s}$, since higher electronic densities tend to screen the external electric field more efficiently. At the same time, the depolarization shift increases with $N_{s}$. As a consequence, the curves of $\Omega(E)$ for different $N_{s}$ are crossing each other. These features are very well reproduced by theory. Ignoring dynamical xc effects (i.e., using RPA) induces a $10 \%$ blueshift of $\Omega$, which then compares less favorably with experiment.

Figure 2 shows the ISB plasmon linewidth $\Gamma(E)$ for different $N_{s}$. For small $E$, the experimental data again exhibit a quadratic behavior, and $\Gamma(E)$ rises faster for smaller $N_{s}$. For large negative $E, \Gamma$ saturates at about $0.7 \mathrm{meV}$. For positive $E$ (i.e., pointing in the direction of sample growth), $\Gamma$ rises somewhat higher. The asymmetry of $\Gamma(E)$ is likely to be due to slightly different roughnesses of the interfaces.

The calculated $\Gamma$ for the clean quantum well (damping through electron-electron interaction only) lies clearly

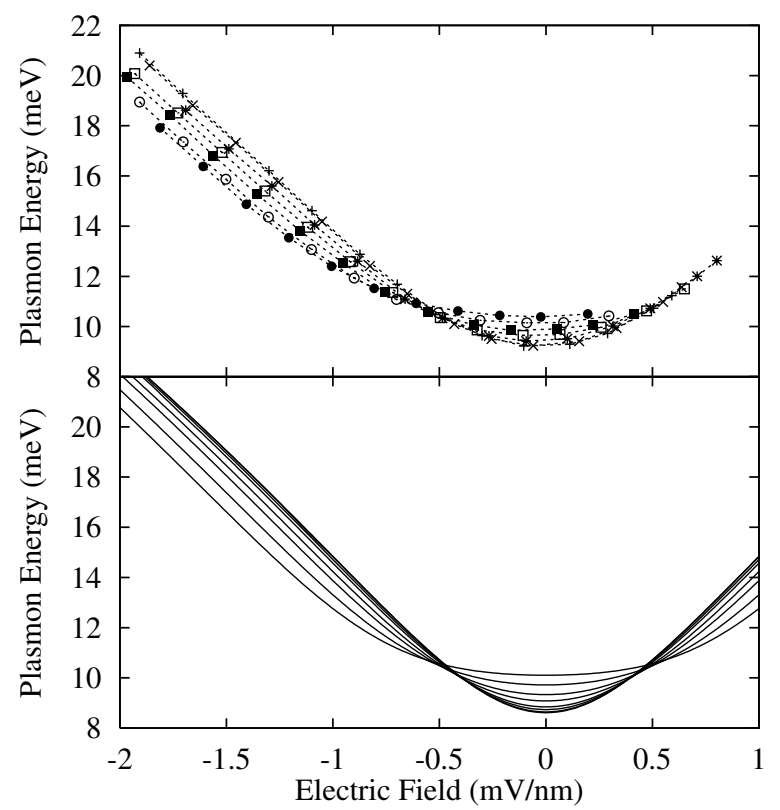

FIG. 1. ISB plasmon frequency versus electric field. Top: experimental data from Ref. [5]. Bottom: calculation for the clean quantum well. The individual curves are for different values of $N_{s}$. At zero field, from bottom to top: $(0.05,0.1,0.2,0.3,0.5$, $0.7,1.0$, and 1.3$) \times 10^{11} \mathrm{~cm}^{-2}$. 


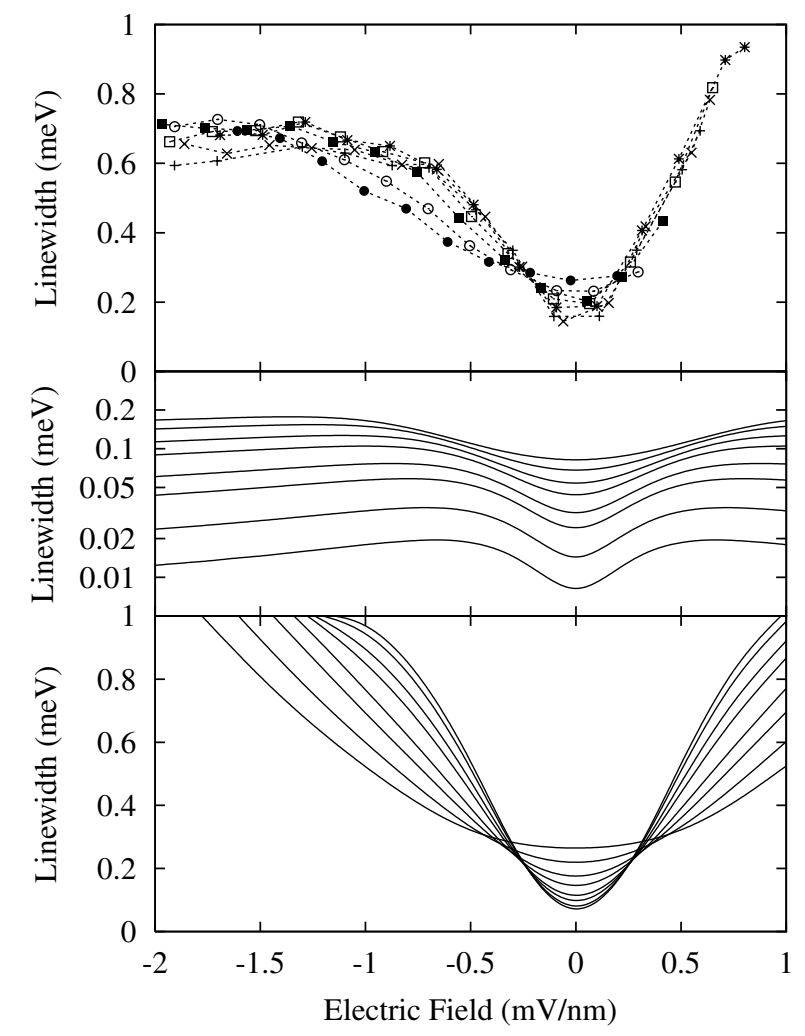

FIG. 2. ISB plasmon linewidth versus electric field. Top: experimental data from Ref. [5]. Middle: calculated results for the clean quantum well. Bottom: the same, but now including impurities and interface roughness. The individual curves are for the same values of $N_{s}$ as in Fig. 1.

below the experimental values, which is hardly surprising. However, it can be seen that these purely electronic effects are far from negligible, at least for $N_{s}$ not too small, and provide an intrinsic lower limit to the linewidth of about 0.1 to $0.2 \mathrm{meV}$ for $N_{s} \sim 10^{11} \mathrm{~cm}^{-2}$.

For a quantitative description of plasmon damping, we now include scattering by charged impurities and interface roughness using the memory function formalism [11-13]. We first briefly summarize the method for homogeneous systems. Consider the correlation functions

$$
C_{i j}(\mathbf{q}, \omega)=\left(A_{i}(\mathbf{q})\left|(\mathcal{L}-\omega)^{-1}\right| A_{j}(\mathbf{q})\right)
$$

between orthonormal variables $A_{i}(\mathbf{q}), \quad\left(A_{i} \mid A_{j}\right)=\delta_{i j}$. Here, $\mathcal{L}$ is the Liouvillian of the system [for definitions of $\mathcal{L}$ and the scalar product $(\cdots \mid \cdots)$, see [11-13]). The correlation functions are coupled by a set of equations:

$$
\sum_{h}\left[\omega \delta_{i h}-\Omega_{i h}(\mathbf{q})+M_{i h}(\mathbf{q}, \omega)\right] C_{h j}(\mathbf{q}, \omega)=-\delta_{i j} .
$$

Here, $\Omega_{i j}(\mathbf{q})=\left(A_{i}(\mathbf{q})|\mathcal{L}| A_{j}(\mathbf{q})\right)$ is the restoring force matrix, and the memory function kernels are

$$
M_{i j}(\mathbf{q}, \omega)=\left(F_{i}(\mathbf{q})\left|(Q \mathcal{L} Q-\omega)^{-1}\right| F_{j}(\mathbf{q})\right),
$$

where $Q$ projects perpendicular to phase-space fluctuations of wave vector $\mathbf{q}$, and $F_{i}(\mathbf{q})$ is the fluctuating force. The correlation functions of interest involve the density fluctuations $A_{0}(\mathbf{q})=\rho(\mathbf{q})(\rho(\mathbf{q}) \mid \rho(\mathbf{q}))^{-1 / 2}$ and the longitudinal current density $A_{1}(\mathbf{q})=j(\mathbf{q}) / \sqrt{n}$, where $n$ is the uniform density of the system. A standard approach for solving Eq. (9) is to set the memory function matrix to zero, except for the diagonal elements:

$$
M_{i j}(\mathbf{q}, \omega)=\left(1-\delta_{i 0}\right) \delta_{i j} M(\mathbf{q}, \omega)
$$

( $M_{00}$ vanishes due to the continuity equation). This leads to the following expression for the density-density correlation function $\Phi(\mathbf{q}, \omega)=C_{00}(\mathbf{q}, \omega) \chi_{c}(\mathbf{q}, 0)[13,15]$ :

$$
\Phi(\mathbf{q}, \omega)=\frac{\chi_{c}(\mathbf{q}, 0) \Phi_{c}(\mathbf{q}, \omega+M(\mathbf{q}, \omega))}{\chi_{c}(\mathbf{q}, 0)+M(\mathbf{q}, \omega) \Phi_{c}(\mathbf{q}, \omega+M(\mathbf{q}, \omega))},
$$

where $\Phi_{c}$ is the correlation function for the clean system. The homogeneous response function is finally obtained via $\Phi(\mathbf{q}, \omega)=\left[\chi(\mathbf{q}, \omega)-\chi_{c}(\mathbf{q}, 0)\right] / \omega$.

Equation (12) is suitable to describe transport phenomena where electronic motion takes place in a homogeneous system, such as the $x-y$ plane of a quantum well. In the case of ISB plasmons, currents are flowing perpendicular to the quantum well. Equation (12) thus has to be generalized to describe the strong inhomogeneity in the $z$ direction due to the interfaces. We summarize here only the results, details will be given elsewhere. Equation (9) becomes

$$
\sum_{h} \int d z^{\prime \prime}\left[\omega \delta\left(z^{\prime \prime}-z\right) \delta_{i h}-\Omega_{i h}\left(\mathbf{q}_{\|}, z, z^{\prime \prime}\right)+M_{i h}\left(\mathbf{q}_{\|}, z, z^{\prime \prime}, \omega\right)\right] C_{h j}\left(\mathbf{q}_{\|}, z^{\prime \prime}, z^{\prime}, \omega\right)=-\delta_{i j} \delta\left(z-z^{\prime}\right)
$$

We again use Eq. (11) and set all off-diagonal elements of $M_{i h}\left(\mathbf{q}_{\|}, z, z^{\prime \prime}, \omega\right)$ equal to zero. Unfortunately, no closedform solution similar to (12) exists, so that Eq. (13) has to be solved numerically. The full response function needed in Eq. (1) then follows from

$$
\begin{aligned}
\Phi\left(\mathbf{q}_{\|}, z, z^{\prime}, \omega\right) & =\int d z^{\prime \prime} C_{00}\left(\mathbf{q}_{\|}, z, z^{\prime \prime}, \omega\right) \chi_{c}\left(\mathbf{q}_{\|}, z^{\prime \prime}, z^{\prime}, 0\right) \\
& =\left[\chi\left(\mathbf{q}_{\|}, z, z^{\prime}, \omega\right)-\chi_{c}\left(\mathbf{q}_{\|}, z, z^{\prime}, 0\right)\right] / \omega .
\end{aligned}
$$

All that remains is to specify the memory function $M\left(\mathbf{q}_{\|}=\right.$ $\left.0, z, z^{\prime}, \omega\right)$, defined as a correlation function between flucapproximation [13], the memory function for chargedimpurity scattering is found to be

$$
\begin{aligned}
M_{I}\left(z, z^{\prime}, \omega\right)= & \int \frac{d^{2} p_{\|}}{\varepsilon^{2}\left(p_{\|}\right)} \frac{\Phi\left(\mathbf{p}_{\|}, z, z^{\prime}, \omega\right)}{\sqrt{n(z) n\left(z^{\prime}\right)}} \int d \tilde{z} n_{i}(\tilde{z}) \\
& \times \operatorname{sgn}(z-\tilde{z}) \operatorname{sgn}\left(z^{\prime}-\tilde{z}\right) \\
& \times e^{-p_{\|}|z-\tilde{z}|} e^{-p_{\|}\left|z^{\prime}-\tilde{z}\right|},
\end{aligned}
$$

where $n_{i}(z)$ is the number of impurities per volume, $\varepsilon\left(p_{\|}\right)$ accounts for dielectric screening [16], and $n(z)$ is the electronic ground-state density in the well. Likewise, the tuating longitudinal forces. In the usual decoupling 
memory function associated with interface roughness is

$$
\begin{aligned}
M_{R}\left(z, z^{\prime}, \omega\right)= & \int \frac{d^{2} p_{\|}}{(2 \pi)^{2}} \frac{\Phi\left(\mathbf{p}_{\|}, z, z^{\prime}, \omega\right)}{\sqrt{n(z) n\left(z^{\prime}\right)}}\left\langle U\left(p_{\|}\right)^{2}\right\rangle \\
& \times \nabla_{z} \nabla_{z^{\prime}}\left[\delta\left(z-z_{l}\right) \delta\left(z^{\prime}-z_{l}\right)\right. \\
& \left.\quad+\delta\left(z-z_{r}\right) \delta\left(z^{\prime}-z_{r}\right)\right],
\end{aligned}
$$

where $U\left(p_{\|}\right)$is the random roughness scattering potential, assumed for simplicity to be the same at the left and right interfaces, $z_{l}$ and $z_{r}$, respectively. The total memory function is the sum of $M_{I}$ and $M_{R}$, and explicitly depends on $\Phi$. This means that $\Phi$ has to be calculated self-consistently, which is a complicated computational task. For simplicity, we use instead the noninteracting $\Phi_{0}\left(\mathbf{q}_{\|}, z, z^{\prime}, \omega\right)$ in (15) and (16), defined by replacing $\chi$ and $\chi_{c}$ with $\chi_{0}$ in Eq. (14). This is expected to be a good approximation as long as plasmon damping is not too strong.

The presence of bulk impurities in the quantum well is mainly caused by segregation of donors from the lower delta-doped layer and diffusion along $z$ during growth. We use the functional form $n_{i}(z)=1.33 e^{-z / 30 \mathrm{~nm}} \times$ $10^{15} \mathrm{~cm}^{-3}$, proposed in Ref. [5] to explain in-plane mobility data, for the bulk impurity concentration in Eq. (15). We also include scattering from the upper delta-doped layer (remote impurity density $4.8 \times 10^{11} \mathrm{~cm}^{-2}$ ).

To describe interface roughness, we take a Gaussian form for the autocorrelation function of the random interface roughness potential [16,17],

$$
\left\langle U\left(p_{\|}\right)^{2}\right\rangle=\pi \mu^{2} \Delta^{2} \eta^{2} e^{-p_{\|}^{2} \eta^{2} / 4} .
$$

Here, $\mu$ is the depth of the quantum well $(257.6 \mathrm{meV}$ for $\mathrm{GaAs} / \mathrm{Al}_{0.3} \mathrm{Ga}_{0.7} \mathrm{As}$ ), and the correlation length $\eta$ and average roughness height $\Delta$ are controlled by material and growth conditions. We find that $\Gamma$ depends mainly on the product $\Delta \eta$ and only little on the particular functional form of $\left\langle U\left(p_{\|}\right)^{2}\right\rangle$.

The bottom part of Fig. 2 shows $\Gamma(E)$ calculated including electronic, impurity, and interface roughness damping. The roughness parameters, $\eta=64.4 \AA$ and $\Delta=4 \AA$, chosen to give the best fit to experiment for the largest $N_{s}$, are in the characteristic range found by lattice imaging techniques [18]. Our results are in good agreement with experiment even away from flat band, as long as $E$ is not too large. For $|E| \geqslant 1 \mathrm{mV} / \mathrm{nm}$, the experimental linewidth saturates, while the calculated linewidth keeps growing. The main reason for this difference is the above-mentioned neglect of self-consistency in the memory functions, as will be discussed elsewhere.

The behavior of $\Gamma$ is dominated by interface roughness scattering and can in fact be qualitatively explained by it alone: Via (16), $\Gamma$ depends on the product of density fluctuations at the edges, which, for $E=0$, have the largest amplitude for highest $N_{s}$. For finite $E$, electrons get pushed towards one edge, but less so for higher densi- ties due to screening of the external field. $\Gamma(E)$ thus rises more steeply for smaller $N_{s}$, and the curves cross.

In Ref. [5] the mobility was found to be dominated by bulk impurity scattering. By contrast, we find that $\Gamma$ is hardly affected by impurities, but is strongly related to interface roughness. However, including electron-electron scattering does lead to a significant quantitative improvement for $\Gamma$, in particular for small $E$.

The theory of the ISB plasmon linewidth presented here allowed us to treat both intrinsic and extrinsic damping mechanisms from first principles and on equal footing. Using reasonable values for the roughness parameters, we obtained quantitative agreement with the experimentally measured linewidth. A remarkable outcome of this study is that the ISB plasmon linewidth is primarily controlled by interfacial roughness, and only weakly affected by the concentration of bulk impurities. The opposite is true for the in-plane mobility, which is primarily controlled by bulk impurities. Thus, the correlation between ISB plasmon linewidth and in-plane mobility is rather weak, which is physically understandable since currents are flowing perpendicular to the quantum well in the former case, and parallel to it in the latter.

This work was supported by NSF Grants No. DMR9706788 and No. DMR-0074959. We acknowledge useful discussions with Jon Williams and Mark Sherwin.

[1] Intersubband Transitions in Quantum Wells I and II, edited by H. C. Liu and F. Capasso, Semiconductors and Semimetals Vols. 62 and 66 (Academic Press, San Diego, 2000).

[2] C. L. Cates et al., Physica (Amsterdam) 2E, 463 (1998).

[3] J. Faist et al., Science 264, 553 (1994).

[4] M. Helm, in Intersubband Transitions in Quantum Wells I (Ref. [1]), Vol. 62, p. 1.

[5] J. B. Williams et al., preceding Letter, Phys. Rev. Lett. 87, 037401 (2001).

[6] K. L. Campman et al., Appl. Phys. Lett. 69, 2554 (1996).

[7] E. K. U. Gross and W. Kohn, Adv. Quantum Chem. 21, 255 (1990).

[8] J. F. Dobson et al., J. Phys. C 21, L729 (1988).

[9] G. Vignale et al., Phys. Rev. Lett. 79, 4878 (1997).

[10] C. A. Ullrich and G. Vignale, Phys. Rev. B 58, 15756 (1998).

[11] D. Forster, Hydrodynamic Fluctuations, Broken Symmetry, and Correlation Functions (Benjamin, Reading, MA, 1975).

[12] F. Yoshida and S. Takeno, Phys. Rep. 173, 301 (1989).

[13] W. Götze, Philos. Mag. B 43, 219 (1981); A. Gold and W. Götze, Phys. Rev. B 33, 2495 (1986).

[14] A. Pinczuk and G. Abstreiter, in Light Scattering in Solids V, edited by M. Cardona and G. Guntherod, Topics in Applied Physics Vol. 66 (Springer, Berlin, 1989), p. 153.

[15] D. Belitz and S. Das Sarma, Phys. Rev. B 34, 8264 (1986).

[16] T. Ando et al., Rev. Mod. Phys. 54, 437 (1982).

[17] G. Fishman and D. Calecki, Phys. Rev. Lett. 62, 1302 (1989).

[18] A. Ourmazd et al., Phys. Rev. Lett. 62, 933 (1989). 